\title{
EVALUACIÓN DE LA CALIDADY EL COSTO DEL ENSILAJE DE PULPA DE NARANJA ELABORADO EN BOLSAS DE POLIETILENO
}

\author{
Jorge L. Morales González¹, Argerie Cruz Méndez¹, Vidal Acuña Redondo, \\ Fernando Dobles Gutiérrez ${ }^{1}$, José L. Bolaños González²
}

RESUMEN

\begin{abstract}
En Costa Rica hay una gran disponibilidad de pulpa de naranja derivada de la industria extractora de jugo. Estos residuos se procesan y utilizan de diferentes maneras en la alimentación de rumiantes. El presente trabajo tuvo como objetivo estudiar el procesamiento de la pulpa en forma de ensilaje y su potencial comerciable al ensilarse en bolsas de polietileno, en términos de calidad (\% proteína cruda - PC) y caducidad (contenido de PC en el tiempo). También se determinó la concentración de fibra neutro detergente (FND) y fibra ácido detergente (FAD) del ensilaje. Se prepararon 54 fardos de ensilaje de pulpa de naranja en bolsas de polietileno, con un peso aproximado de $45,00 \mathrm{~kg}$ cada una. Las bolsas con la pulpa fueron tratadas con 0,75 y $150 \mathrm{~g}$ de urea por bolsa; cada nivel de urea se replicó tres veces. El ensilaje se muestreó para análisis químico a los $30,45,60,180,360$ y 540 días después de elaborado. Los datos fueron analizados en un diseño de bloques al azar con arreglo de parcelas divididas. El ensilaje con el nivel de 150,00 g de urea por bolsa, resultó en un aumento de la PC de 6,98 a $11,10 \%(P<0,05)$ y una disminución del 17,90 al $15,6 \%$ de materia seca, con respecto al ensilaje sin urea. Los datos disponibles hasta 360 días indican un contenido de PC de 8,3 a 9,8 \% y buenas características organolépticas, éstas últimas manteniéndose hasta los 540 días del estudio. Los valores y el mayor contenido de FAD, con respecto a FND en el ensilaje, confirma el alto contenido de pectina (fibra neutro detergente soluble) en la pulpa de naranja y su alta digestibilidad. Se concluye que la pulpa de naranja se puede ensilar directamente en bolsa de polietileno, obteniéndose un producto que se puede conservar en buen estado hasta por 1,50 años y cuya PC, se puede mejorar con la adición de $150 \mathrm{~g}$ de urea/ $45 \mathrm{~kg}$ de pulpa, lo cual aunado a las características de su fibra permiten comercializar un producto de excelente calidad para la alimentación animal.
\end{abstract}

Palabras clave: Residuos de naranja, comercialización, digestibilidad, subproduco.

\section{INTRODUCCIÓN}

Existen diferentes alternativas tecnológicas que podrían mejorar la capacidad de carga de nuestros sistemas de producción de carne y leche bovina y la nutrición en general de nuestros hatos. Por ejemplo, a través de especies forrajeras mejoradas para pastoreo y corte, así como prácticas de manejo animal y de pasturas o a través de la alimentación suplementaria.

En los últimos años ha surgido un subproducto con gran potencial para la suplementación animal, que no está siendo utilizado significativamente y que, por tanto, requiere atención inmediata. Este es la pulpa de naranja, la cual se está produciendo en grandes cantidades en el país, en las plantas extractoras de jugo de naranja (cerca de 200 mil toneladas anuales) ${ }^{3}$, todo esto producto del área creciente ocupada por el cultivo de la naranja, que cubre más de 30000 ha (Araya 2001).

La pulpa de cítricos, está compuesta de 60-65 \% de cáscara, 30-35 \% de pulpa y 0-10\% semillas (Braddock 1999); es

\footnotetext{
1 Instituto Nacional de Innovación y Transferencia en Tecnología Agropecuaria (INTA), Costa Rica. Correo electrónico: jmorales@ inta.go.cr.

2 Exgerente de Producción del Grupo del Oro, Costa Rica. Actualmente Gerente General INARROZ, Costa Rica. Correo electrónico: jbolanos@inarroz.cr.com.

3 Hernández, S. 2008. Producción de pulpa de naranja en plantas extractoras de jugo. Instituto Nacional de Innovación y Transferencia en Tecnología Agropecuaria. Comunicación Personal ( q.d.D.g.).
} 
considerada un concentrado voluminoso alto en energía (74 \% de nutrientes digestibles totales), pero bajo en proteína (6\%). Los animales la consumen en su estado fresco sin procesar ó procesada como ensilaje, pulpa seca o pelets (Wing 2003). Es muy palatable y bien utilizada por los rumiantes cuando no excede el $30 \%$ del total de la materia seca consumida. Además, es una buena fuente de calcio, pero muy baja en caroteno (Blezinger 2003). Como pulpa fresca tiene el inconveniente que su alto contenido de humedad (aproximadamente $80 \%$ ), la hace accesible únicamente para las explotaciones ganaderas cercanas a las plantas de extracción de jugo, ya que el transporte de tales cantidades de agua no sería rentable.

La cáscara contiene aceites, principalmente d-limonina y algunos otros flavones polymetolados como taninos, saponinas, phytatos, oxalatos, flavonoides, conocidos como factores anti-nutricionales los cuales pueden tener efectos negativos en los animales, pero que en el presente caso, por sus bajas concentraciones no representan ningún problema, al contrario se ha observado que por ejemplo, en dietas de rumiantes con contenidos del 2 al $3 \%$ de taninos condensados, éstos ofrecen efectos benéficos porque reducen la degradación de la proteína en el rumen, mediante la formación de una proteína compleja (Oluremi et al. 2007).

La pulpa en forma de pelets y particularmente en forma de pulpa seca se utiliza mucho en la alimentación animal, precisamente porque es sometida a procesos de extracción de humedad, lográndose un producto final mucho más manejable desde el punto de vista de transporte, almacenamiento y para proveerla a los animales en mezcla o directamente en la canoa (Chapman et al. 2008). Sin embargo, fuera de que la peletización puede tener un efecto favorable en la tasa de pasaje ruminal, nutricionalmente no hay ninguna diferencia entre pulpa de naranja peleteada o no (Wing 2003). El alto costo del proceso de secado, podría eventualmente hacer más atractivo el uso de la pulpa húmeda en fresco (Blezinger 2003), ó conservada en forma de ensilaje. En nuestro país también está siendo utilizada, para la alimentación animal en las tres presentaciones mencionadas anteriormente.

Su uso en la alimentación animal, permitirá además, reducir o eliminar, un subproducto que por sus características físico-químicas y por su volumen de producción, podría convertirse en un elemento contaminante. Pero más importante aún y precisamente por las características físico-químicas y de volumen disponible, este subproducto podría contribuir al aumento en la capacidad de carga animal y la nutrición de los sistemas de producción de leche y de carne.

El desarrollo y/o la validación de la tecnología existente, para el tratamiento y el uso de la pulpa de naranja en la alimentación animal es la base del presente estudio. Un método de procesar la pulpa de naranja fresca, que se está utilizando en pequeñísima escala y probablemente a un nivel técnico un poco rudimentario, por lo menos a nivel de Costa Rica, es el ensilaje de pulpa de naranja. Algunas experiencias de productores indican que se obtiene un buen ensilaje y que es una manera de almacenar esta materia prima en la época de excedentes para las épocas de baja disponibilidad de forraje en las fincas, y que se podría mejorar su contenido de proteína utilizando algunos aditivos al ensilar. Una de las empresas extractoras de jugo, ensila grandes cantidades de pulpa al final de la zafra, pero con fines ambientales y de conservación del producto, para continuar la elaboración de abono orgánico en la próxima estación seca.

Técnicamente, el ensilaje parece una forma viable de utilizar este subproducto, sin embargo, el procesamiento se está realizando mediante el silo de trinchera o de montón, lo cual no soluciona el problema del alto contenido de humedad para el transporte y por lo tanto su utilización se restringiría, igualmente, a las áreas aledañas a las plantas extractoras.

Usando el mismo principio del ensilaje, que permite el almacenamiento de alimentos para animales por largas temporadas $y$ 
manteniendo su contenido nutricional, utilizando otros medios diferentes al silo de trinchera y de montón, es el ensilado directo en bolsas de polietileno. Algunas de las ventajas sobre el método convencional son: calidades similares del producto, bajo costo, puede hacerse manualmente, fácil de manipular y de usar, transportable y no ocurren pérdidas nutricionales por efluentes (Ashbell and Weinberg sf; Fraser et al. 2004; Ashbell et al. 2001; Lane 2000; Moran 2005; Morales et al. 2001 y 2004).

El proceso de ensilaje debería lograrse con iguales y hasta mejores resultados en una bolsa de polietileno cuidadosamente sellada; ya que permite añadir aditivos para mejorar la calidad del producto final. Pero más importante, es la manera viable de lograr transportar el alimento a cualquier lugar del país y almacenarlo por largas temporadas, siempre y cuando, las bolsas de polietileno reúnan algunas características mínimas de resistencia para el transporte y el almacenamiento y un tamaño adecuado para que de su contenido se puedan alimentar varios animales, pero que a la vez no sean tan grandes y pesadas que no puedan ser manipuladas por una persona. Basado en las anteriores consideraciones se propuso el presente estudio de investigación cuyo objetivo fue: evaluar la calidad, la caducidad y el costo del ensilaje de pulpa de naranja elaborado en bolsas de polietileno.

\section{MATERIALES Y MÉTODOS}

El presente estudio se llevó a cabo de mayo de 1999 a noviembre del 2000, en la Estación Experimental Enrique Jiménez Núñez (E.E.E.J.N.), perteneciente al INTA, ubicada en San Miguel de Cañas, Guanacaste, 10 $\mathrm{km}$ al sureste del distrito central y $10 \mathrm{~km}$ al sur de la Carretera Panamericana, la cual se encuentra a una altura de $9-11 \mathrm{msnm}$.

Las condiciones agro-ecológicas de la localidad están relacionadas a la Zona de Vida de Bosque Húmedo, transición a Basal y Tropical, caracterizadas por fuertes vientos y un patrón climático bimodal, con un periodo seco de diciembre a abril y un invierno intenso en lluvias, de setiembre a mediados de noviembre. Estos separados por un periodo que se ha manifestado errático en los últimos años, ligero de lluvias de mayo a mediados de julio y de aquí a agosto en que se reduce la precipitación significativamente, correspondiente al veranillo de San Juan y a la canícula. La Figura 1, muestra el comportamiento del clima en la zona (Hancock y Hargreaves 1977), la cual está basada en un periodo de seis años y con probabilidades de un $75 \%$ de que este patrón de lluvias pueda ocurrir nuevamente.

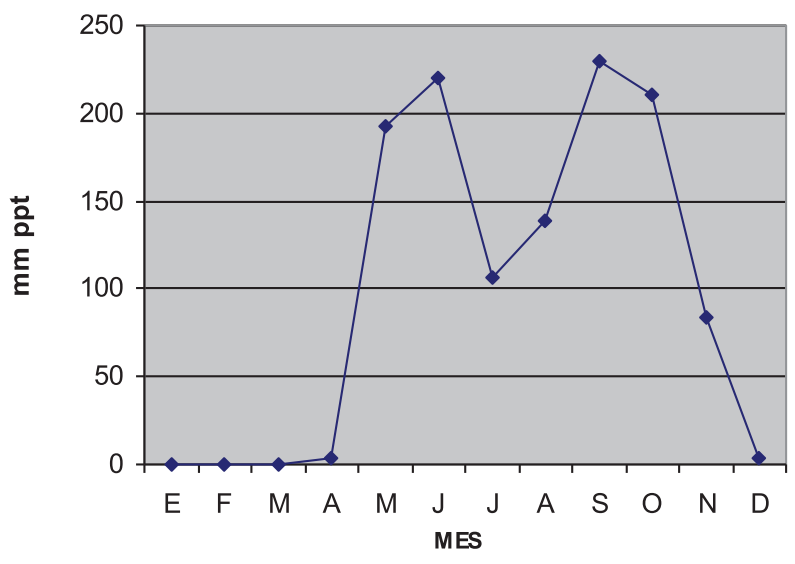

Figura 1. Patrón de lluvias con $75 \%$ probabilidades para el área de la E.E.E.J.N. Guanacaste, Costa Rica. 2000.

La temperatura promedio de la zona ha sido de 24,60ㄷ; la humedad relativa $91 \%$; precipitación máxima de $2818 \mathrm{~mm}$; precipitación mínima de $1018 \mathrm{~mm}$, durante el mismo periodo.

La materia prima, i.e., la pulpa de naranja, fue facilitada por la empresa "Del Oro", ubicada en Santa Cecilia, del cantón de La Cruz en Guanacaste. El procesamiento se realizó en la propia planta extractora de jugo y el almacenaje y muestreo del producto se hizo en la E. E. E. J. N. 


\section{Procesamiento}

Bolsas. En esta prueba se utilizaron bolsas de polietileno de $60 \mathrm{~cm}$ de ancho por 110 $\mathrm{cm}$ de largo, de 5 micras de grosor en ambas caras. Se seleccionó este tipo de bolsa porque facilita la carga, transporte, almacenamiento y manipulación ulterior, de un fardo con un contenido de $45 \mathrm{~kg}$ de pulpa de naranja, cuyas características físicas de humedad y maleabilidad, hacen difícil su manipulación de otra manera. Esta es condición necesaria, además del valor nutricional, para que un producto para la alimentación animal como éste reúna las características para ser comercializado, como se pretendió en el presente estudio.

Manejo. La pulpa de naranja fresca, depositada por vagonetas en el suelo, cerca de la planta extractora de jugo, fue tomada con pala e introducida en la bolsa plástica que se calibró, al peso deseado de $45 \mathrm{~kg}$. Simultáneamente con cada palada se aplicó, utilizando una botella plástica, la urea previamente disuelta en la menor cantidad de agua que permitió su disolución, para no aumentar más, el ya de por sí, alto contenido de humedad de esta materia prima.

Cada bolsa se agitó repetidamente, para lograr una compactación interna adecuada, extraer todo el aire posible y dar las condiciones anaeróbicas requeridas para un ensilado adecuado. Afortunadamente, las características naturales de la pulpa de naranja permiten esa condición fácilmente con la manipulación mencionada. Inmediatamente después, se procedió a retorcer la boca de la bolsa manualmente, hasta que quedó bien apretada y amarrarla con mecate plástico (piola). Luego, cada bolsa se identificó e introdujo en sacos de propileno, los cuales permiten reforzar aún más la capacidad de manipulación señalada anteriormente, i.e., sin dañar el fardo y deteriorar el producto.

En total se elaboraron 54 bolsas de $45 \mathrm{~kg}$ cada una, conformando tres grupos de 18, los cuales fueron tratados con: 0,75 y $150 \mathrm{~g}$ de urea, respectivamente.
Tratamientos. Uno de los nutrientes básicos en la alimentación de rumiantes, es la proteína. Precisamente este nutriente es bajo en la pulpa de naranja, con sólo un $6 \%$ en base seca, si se toma como referencia que el contenido de 6-8 \% de proteína en pasturas, se considera el mínimo antes de que el consumo de forraje por el animal se reduzca drásticamente (Minson 1981).

En nutrición de rumiantes se habla de la proteína como proteína cruda (PC), ya que este tipo de animales herbívoros cuentan con un sistema digestivo complejo, del cual, uno de sus principales compartimientos es el rumen; que con sus características físicas, fisiológicas y de simbiosis con una rica microfauna y flora, hace posible la utilización, además de alimentos fibrosos, de fuentes no proteicas de nitrógeno (NNP), convertibles en fuentes de nutrientes energéticos y de proteína verdadera, respectivamente, para el animal.

Dado que la producción de ensilaje bajo las condiciones realizadas en el presente estudio, permiten agregar aditivos para mejorar su estatus nutricional, se optó por esta posibilidad mediante el tratamiento de la pulpa de naranja con urea, fuente de nitrógeno no proteico utilizable por los rumiantes. Se reconoce que la pulpa de cítricos puede contribuir a una mejor utilización del amonio y por tanto de fuentes proteicas y de nitrógeno no proteico como la urea a nivel del rumen (Wing 2003). Se considera que en el caso de leche, la máxima producción ocurre cuando la proteína degradable a nivel de rumen es del 12,2\% de la dieta en base seca (NRC 2001), i.e., el nivel máximo de nitrógeno no proteico utilizable a nivel ruminal es el de su valor convertido a un máximo de $12 \%$ de proteína cruda, nivel después del cual se requiere proteína verdadera de baja degradabilidad ruminal, si se buscan respuestas mayores de producción de leche o carne, en animales con dicho potencial de respuesta.

Con base en los valores de conversión de nitrógeno a proteína cruda $(\mathrm{PC}=\mathrm{N} \times 6,25)$, 
(NRC 2001), el contenido de nitrógeno de la urea (45\%), el nivel natural de PC de la pulpa de naranja (6\%) y el máximo de $12 \%$ de PC basado principalmente en NNP (nitrógeno no proteico), aprovechable para la producción por el rumiante, se calculó que tratando los 45 $\mathrm{kg}$ de pulpa de naranja con $150 \mathrm{~g}$ de urea, se alcanzaría ese máximo de $12 \%$ PC. Además y dado que entre las incógnitas estaba el efecto del proceso de ensilado sobre ese resultado esperado de $12 \%$ de PC y que se preparaban cantidades mayores de ensilaje simultáneamente para pruebas con animales, lo cual aumentaba aún más la incógnita de resultados esperados, se optó por un rango amplio de niveles de 0,75 y $150 \mathrm{~g}$ de urea para poder disponer de un margen amplio de contenidos de PC, que como tratamientos permitieran una mayor posibilidad de observar respuestas y generar conclusiones más precisas y prácticas.

Basado en las anteriores consideraciones, los tratamientos aplicados fueron, por tanto:

1.- Pulpa de naranja sin urea.

2.- Pulpa de naranja con $75 \mathrm{~g}$ de urea.

3.- Pulpa de naranja con $150 \mathrm{~g}$ de urea.

Con estos niveles de aplicación de urea, se esperaría obtener ensilajes con contenidos de 6,9 y $12 \%$ de proteína cruda, respectivamente.

Fases de evaluación y muestreos. Dentro del concepto de calidad de un ensilaje, también es importante determinar durante cuánto tiempo puede mantenerse dicha calidad, ya que ésta es una condición fundamental para la comercialización, transporte y almacenamiento, tanto en planta como en finca, de un producto como el que se desarrolló en este estudio. Por esto se realizaron, dos pruebas:

\section{Procesamiento}

Se determinó cual es el tiempo mínimo necesario para que el proceso anaeróbico produzca la mejor calidad de ensilaje. Se usaron como referencia los tiempos de
30, 45 y 60 días que requiere el ensilaje de otros materiales como el maíz, para aplicarlos en el tipo de ensilaje objeto del estudio.

\section{Caducidad}

Se determinó hasta cuándo podría utilizarse el producto sin tener pérdidas significativas en calidad. Para esto se asumió que si un producto como el presente durara año y medio sin perder sus características nutricionales, se rebasarían ampliamente los factores críticos de utilización y comercialización relacionados con el tiempo de almacenamiento, por lo que se aplicaron los siguientes tiempos: 180, 360 y 540 días.

\section{Diseño Experimental}

El estudio incluyó tres tratamientos basados en tres niveles de urea y seis momentos de muestreo en el tiempo, con tres repeticiones. Las repeticiones utilizadas corresponden a los bloques, según la posición a nivel del suelo, de las pilas de tres bolsas que se hicieron en el almacenaje, para dar los periodos de fermentación anaeróbica ó caducidad. El arreglo de los bloques fue el siguiente:

Bloque 1: Tratamientos a nivel del piso.

Bloque 2: Tratamientos colocados sobre el bloque 1 .

Bloque 3: Tratamientos colocados sobre el bloque 2 .

El diseño experimental utilizado fue de Bloques al Azar con arreglo de parcelas divididas, para ambas pruebas, i.e., procesamiento y caducidad, según el siguiente ANDEVA (Cuadro 1): 
Cuadro 1. Análisis de varianza. Guanacaste, Costa Rica. 1999

\begin{tabular}{cc}
\hline Fuente de variación & Grados de libertad \\
\hline BLQ & $3-1=2$ \\
DP & $6-1=5$ \\
BLQ*DP error a & $5 \times 2=10$ \\
GRU & $3-1=2$ \\
GRU*DP & 10 \\
Residuo error b & 24 \\
\hline Total & $\mathbf{5 4 - 1 = 5 3}$
\end{tabular}

Parcela principal $=$ días de procesamiento (DP) ó caducidad (CD)

Sub-parcela $=$ niveles de urea $(G R U)$

\section{Variables dependientes}

Las variables dependientes fueron los parámetros de calidad del ensilaje basados en porcentaje de: materia seca (MS), proteína cruda (PC), fibra neutro detergente (FND) y fibra ácido detergente (FAD).

Se intentó analizar el contenido de nitrógeno amoniacal del ensilaje, el cual se considera como uno de los parámetros más adecuados para determinar la calidad de los ensilajes. Sin embargo no fue posible hacer la extracción de líquidos de la muestra, paso fundamental de la metodología, ya que la presión que se ejerce para hacer la extracción, por las características del ensilaje de pulpa de naranja, no produjo la separación de la parte líquida de la sólida, sino que ambas fases pasaron la malla que debería permitir tal separación.

\section{Muestreos}

Al cumplirse los diferentes tiempos previstos para determinar calidad del ensilaje, se tomaron muestras compuestas de cada saco, utilizando un dispositivo de metal con punta afilada, diseñado con este propósito, que permitió muestrear la pulpa de cualquier sitio del saco. Se logró muestrear todos los tratamientos y sus repeticiones hasta el periodo de 360 días. De las muestras correspondientes al periodo de 540 días (1,5 años), sólo se pudo muestrear una repetición del tratamiento de $150 \mathrm{~g}$ de urea, ya que el resto fue deteriorado por ratas, el cual es un problema a considerar durante el almacenamiento.

\section{Registro de costos}

Todos los costos de la elaboración del ensilaje se registraron para determinar el costo del producto final.

\section{Análisis de Laboratorio}

En el laboratorio de Piensos y Forrajes del INTA, muestras frescas de alrededor de $1 \mathrm{~kg}$ de ensilaje de pulpa, previamente pesadas en balanza granataria, e secaron en estufa a 60 C durante $72 \mathrm{~h}$, se tomaron los pesos secos y se molieron en molino de martillos utilizando malla de $2 \mathrm{~mm}$. Posteriormente estas muestras se secaron en horno a $105^{\circ} \mathrm{C}$ durante 24 horas, para determinar materia seca. Las muestras se analizaron para contenido de proteína (\% PC), utilizando el método de microKjeldahl, \% de fibra ácido detergente (FAD), (AOAC 1980) y fibra neutro detergente \% FND utilizando la metodología de Van Soest (Goering and Van Soest 1970).

\section{RESULTADOS}

\section{Contenido de materia seca}

La pulpa fresca de cítricos presenta contenidos de materia seca del 20 al $23 \%$ (Wing 2003 y Moran 2005), sin embargo, en el ensilaje de pulpa de naranja, estos contenidos fueron aún más bajos, i.e de 17,40 hasta 16,60. Esto debido a la alta humedad natural de la pulpa de naranja y al periodo de almacenamiento, que después de un año alcanza su nivel más bajo $(P<0,10)$ (Figura 1$)$. 


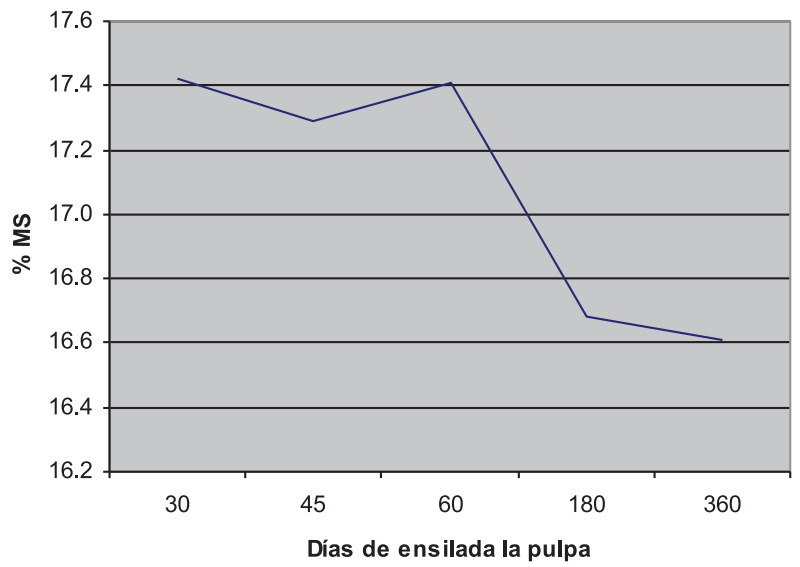

Figura 2. Contenido de materia seca del ensilaje de pulpa de naranja durante diferentes periodos de almacenamiento. Guanacaste, Costa Rica. 1999.

La cantidad de urea aplicada fue un factor importante también en la reducción del contenido de materia seca del ensilaje $(P<0,001)$. Hasta $75 \mathrm{~g}$ de urea aplicada el ensilaje mantuvo un $18 \%$ de materia seca, posteriormente ésta se redujo significativamente, hasta cerca del $15 \%$ cuando se aplicaron $150 \mathrm{~g}$ de urea (Figura 3).

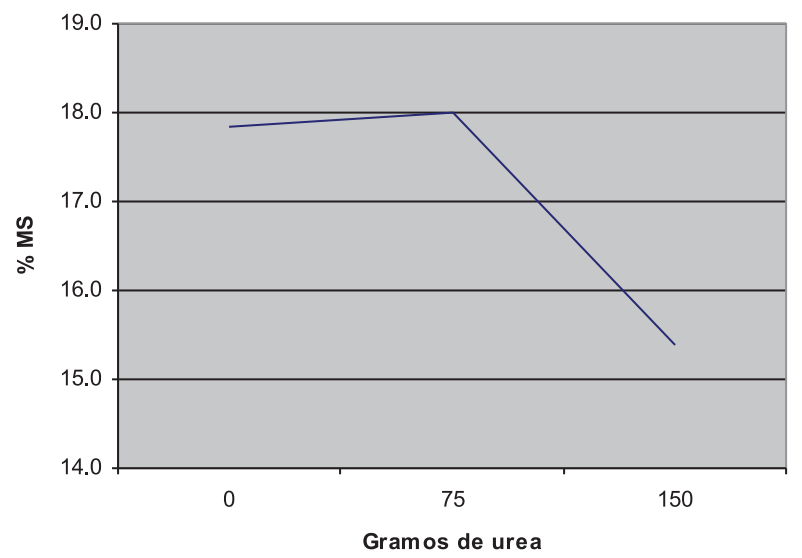

Figura 3. Contenido de materia seca del ensilaje de pulpa de naranja según cantidad de urea utilizada. Guanacaste, Costa Rica. 1999.

Afortunadamente, el ensilado directo en bolsas plásticas, no permite, como sí es el caso en los sistemas tradicionales, la pérdida de nutrientes por efecto de drenado, particularmente cuando materiales con alta humedad se ensilan, como ocurre con el ensilado de la pulpa de naranja en sistemas tradicionales con pérdidas de más de un $50 \%$ de la materia seca, por los grandes volúmenes de efluentes (Ashbell y Weinberg sf). Por tanto, mediante este método de ensilaje se conserva mejor la calidad de la pulpa de naranja y también hay un impacto ambiental negativo que se soluciona con el ensilado directo en bolsas plásticas, las cuales además son reciclables.

\section{Contenido de proteína cruda}

El ensilaje de pulpa de naranja presentó un aumento significativo $(P<0,05)$ en su contenido de proteína cruda, en el tiempo. El aumento fue de más de una unidad porcentual de los 30 a los 360 días de ensilado (Figura 4). Este efecto debe estar relacionado, con la disolución y entrapamiento progresivo de la urea en todo el material ensilado conforme el periodo de almacenamiento se fue prolongando.

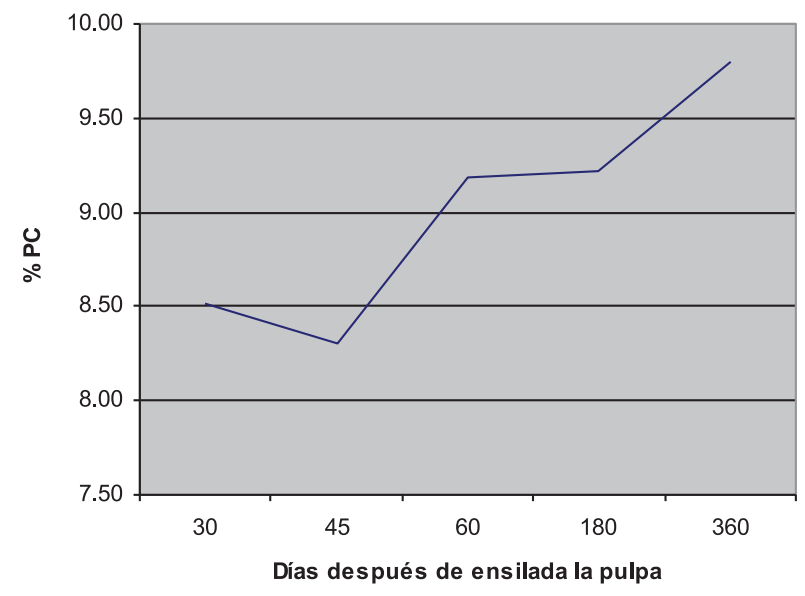

Figura 4. Contenido de proteína en el ensilaje de pulpa de naranja durante el periodo de almacenamiento. Guanacaste, Costa Rica. 1999.

En un estudio similar en donde se aplicó urea, pero en maíz (Weiss 2008), por sus bajos niveles de proteína ( $8 \%$ en base seca), se aplicaron entre 4,50-6,40 kg de urea/t de forraje, niveles superiores al utilizado en el presente trabajo, se logró incrementar la PC del ensilaje en 2,60 unidades porcentuales. En el presente estudio (Figura 4), se lograron aumentos mayores, de 
hasta cuatro unidades porcentuales, diferencia debida probablemente a que en el caso del maíz el silo permitía la pérdida de fluidos por drenaje, lo cual no sucede con el ensilaje en bolsa plástica.

Aunque se ha comparado el contenido de energía y proteína de la pulpa de naranja con el del maíz (Oluremi et al. 2007), el hecho es que la pulpa de naranja, es un subproducto agroindustrial alto en energía digestible para rumiantes, pero con un contenido natural de proteína bajo (Blezinger 2003). Con un contenido de alrededor del $7 \%$ de proteína cruda según el presente estudio (Figura 5), este alimento apenas llena los requisitos mínimos que se consideran necesarios, para que un rumiante obtenga las necesidades mínimas nutricionales para la producción (Minson 1981). Aunque este no es un problema, pues este alimento sería apenas parte de una dieta más completa para alimentación de ganado semi o estabulado, en el caso de ser utilizada como suplemento en épocas críticas de escases de forrajes en cantidad y calidad, el aditivo es esencial.

Por ello, es tarea obligada hacer uso de la ventaja del ensilado en bolsas de polietileno, para agregar urea y así mejorar el contenido de proteína del producto final. La adición de urea en el presente estudio, permitió que la pulpa de naranja ensilada aumentara su contenido de $P C(P<0,0001)$ (Figura 5). Con $75 \mathrm{~g}$ de urea por cada $45 \mathrm{~kg}$ de pulpa se aumentó la PC de 6,98 a $8,85 \%$. Con $150 \mathrm{~g}$ de urea se logró alcanzar $11,20 \%$ en el contenido de PC del ensilaje. Este último nivel es muy aceptable y está dentro del rango que podrían manejar los animales para producir, sin peligro de intoxicaciones por excesos de urea en la ingesta. Resultados similares fueron obtenidos en otros estudios, con pulpa de cítricos tratada con amonio líquido en donde los animales consumieron hasta en un $30 \%$ los requerimientos de proteína cruda y en un $50 \%$ las necesidades de energía, en ganado adulto, y en ganado joven, alimentados con pulpa con un $12 \%$ de PC, produjo un crecimiento satisfactorio (Loosli; Mc Donald 1968).

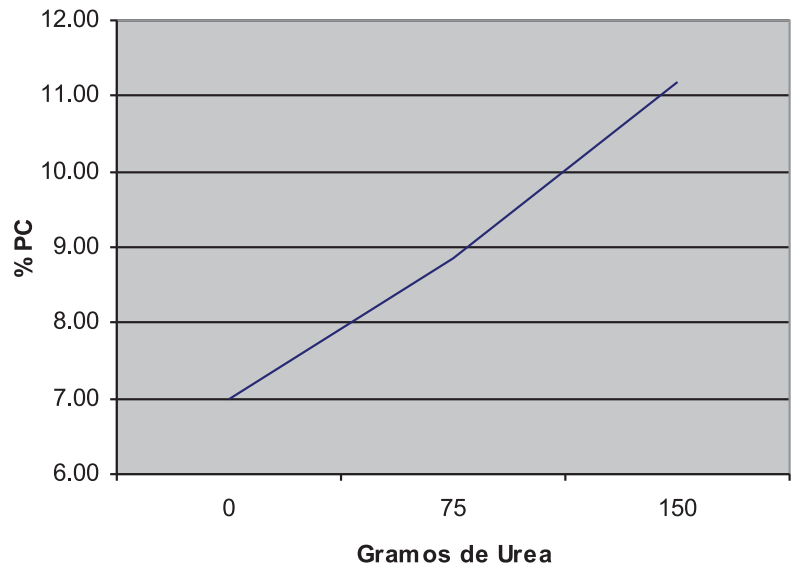

Figura 5. Contenido de proteína del ensilaje de pulpa de naranja según la cantidad de urea utilizada. Guanacaste, Costa Rica. 1999.

\section{Contenido de fibra}

El contenido de fibra de la pulpa de naranja ensilada presenta valores muy diferentes a los otros alimentos para rumiantes, particularmente de aquellos fibrosos como los forrajes. Por ejemplo, el contenido de FND en gramíneas anda sobre el $60 \%$ (Morales et al. 2006 a y b) y en leguminosas sobre el 40 \% (Morales 1989 y Morales et al. 2005), además de que éste es siempre mayor que la FAD (35 a $45 \%$ ), en éstos últimos alimentos mencionados, inclusive hasta en la pulpa de café (Getachew et al. 2005).

El contenido de FND aumentó con el incremento en urea $(P<0,05)$, pasando de 24,60 a 28,10\%, cuando se aplican 0 y $150 \mathrm{~g}$ de urea, respectivamente (Figura 6). 
Morales et al::Evaluación de la Calidad y el Costo del Ensilaje de Pulpa de Naranja elaborado en Bolsas de Polietileno1

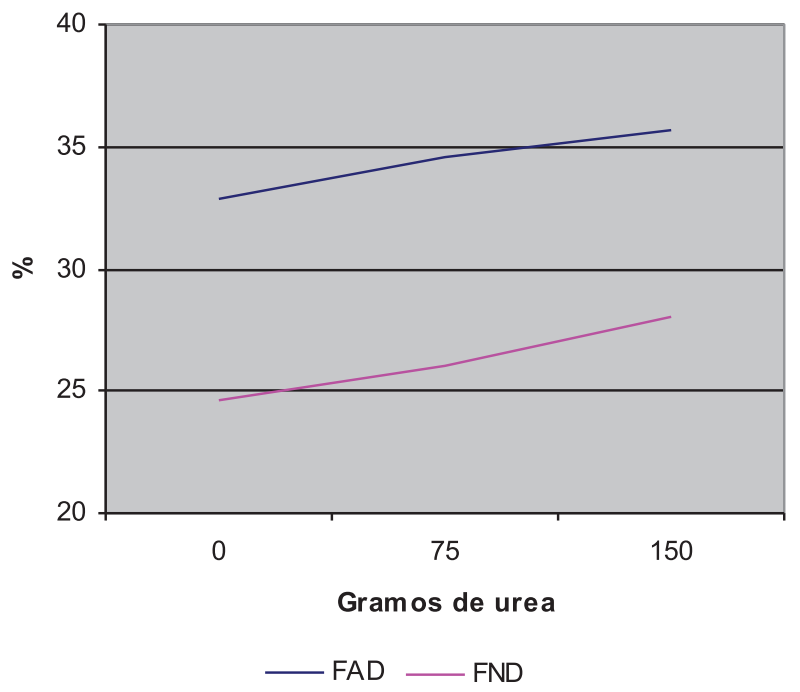

Figura 6. Contenido de fibra del ensilaje de pulpa de naranja según cantidad de urea utilizada. Guanacaste, Costa Rica. 1999.

En el caso de la pulpa de naranja se presenta una situación contraria con un contenido mayor de FAD en relación a la FND (33 y 25 $\%$ promedio, respectivamente). Todos los valores de las fibras detergentes en la pulpa de naranja ensilada, en general, son menores a aquellos encontrados en los forrajes.

En el caso del contenido de FAD, se encontró una interacción entre el tiempo de ensilado y la cantidad de urea aplicada $(P<0,05)$. Sin embargo, este efecto no parece ser de mayor importancia en el contenido de este tipo de fibra en el ensilaje de pulpa de naranja (Figura 7).

Los contenidos de fibra observados en el ensilaje de pulpa de naranja, probablemente estén relacionados con su composición y tipo de polisacáridos no almidonosos, la cual está compuesta principalmente por pectinas, mucilágenos, entre otros, de cadenas cortas y no de celulosas y hemicelulosas de cadena larga como es en el caso de las plantas forrajeras y a la metodología de extracción utilizada en el laboratorio (Van Soest et al. 1991), en la cual el detergente neutro diluye toda la pectina y el detergente ácido no diluye toda la pectina.

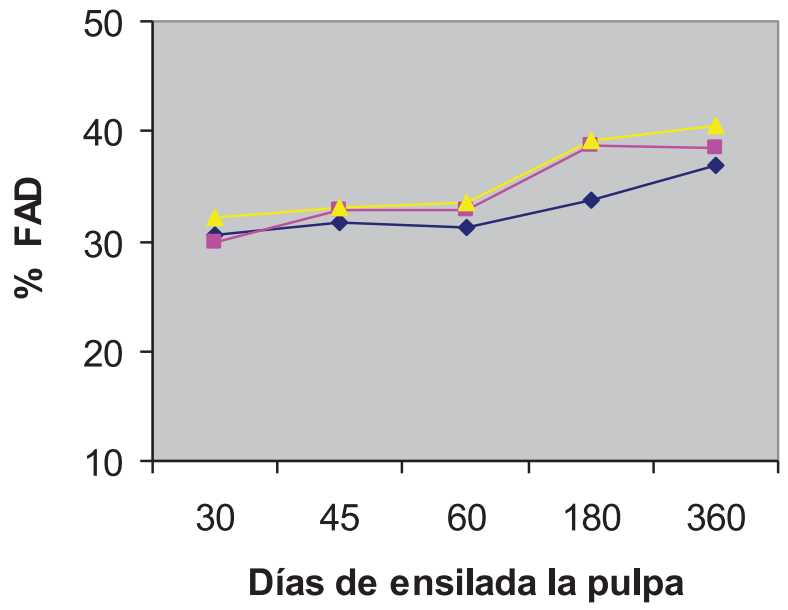

$\multimap 0 \mathrm{~g} \longrightarrow 75 \mathrm{~g} \longrightarrow 150 \mathrm{~g}$

Figura 7. Contenido de fibra ácido detergente del ensilaje de pulpa de naranja a través del tiempo según el nivel de urea aplicada. Guanacaste, Costa Rica. 1999.

Hay dos tipos de paredes celulares en las plantas, la primaria y la secundaria, ésta última generalmente lignificada. La pectina es una familia de polisacáridos complejos y son los principales componentes en las paredes celulares primarias de las plantas. Estas paredes celulares primarias son las primeras paredes en aparecer en todas las células en crecimiento y en división en las plantas. Estas estructuras rodean las células en las partes suaves y comestibles de las plantas y son el tipo de paredes predominantes en frutas y vegetales (Mohnen 2007). Los sólidos en la pulpa de naranja están compuestos por $40 \%$ de azúcares y 50 \% por polisacáridos, estos últimos representados principalmente por sustancias pécticas (Citrech 2008).

La pared celular primaria puede dividirse en dos tipos, tipo I que se encuentra en todas las plantas con inflorescencia con excepción de la familia de las gramíneas y el tipo II que se le encuentra sólo en la familia de las gramíneas (Poaceae). La composición fibrosa de las paredes del Tipo I es igual al del Tipo II, i.e, celulosa, hemicelulosa y pectina, pero con la diferencia de que la proporción de pectina en el Tipo I es del 30 al $35 \%$ y en el Tipo II la proporción de la pectina es de alrededor del 
$10 \%$, así como el tipo de hemicelulosas y pectinas es diferente en una y otra (Mohnen D. sf.).

Por lo tanto, el resultado en el presente estudio indica que las determinaciones de fibra basados en detergentes como el FND y FAD originales (Van Soest 1963), no son comparables entre plantas diferentes como son las leguminosas y las gramíneas, o como en el presente caso entre subproductos de la industrialización de frutas y gramíneas. Este resultado concuerda con las observaciones hechas por Reed y Van Soest (1985), Jung 1997 y Mertens (2003).

La pectina en la pulpa de naranja queda incluida en la fracción soluble neutro detergente, con el método de determinación para FND. Esta fracción junto con los demás componentes solubles al detergente neutro, se estima que tienen una disponibilidad nutritiva uniforme, con una digestibilidad verdadera del 98,00 \% (Reed y Van Soest 1985). Considerando que la FND fue de un 25 $\%$, la pulpa de naranja contiene al menos un $75 \%$ de sólidos digestibles (100-FND $=100$ $25)$, lo que la convierte en un alimento de alta calidad para los animales. La pulpa de cítricos consiste de pectina y celulosa; este material es aproximadamente un $45 \%$ de fibra soluble, la cual representa el componente de pectina de toda la fibra, es decir la pectina es altamente degradada por la microflora ruminal. La desaparición de la materia orgánica, en orden de menor a mayor es según el siguiente orden: celulosa < pulpa de remolacha $<$ pulpa de cítricos < pectina de cítricos, en promedio entre diferentes especies animales incluidos el humano, el ganado, el cerdo, el caballo, el perro y el gato (Sunvold et al. 1995)

De acuerdo con Van Soest et al. (1991), ha ocurrido un considerable desarrollo con los métodos de determinación de fibra en los alimentos, debido a la redefinición de la fibra dietética para el hombre y animales monográstricos, que incluye lignina y todos los polisacáridos resistentes a las enzimas digestivas de los mamíferos. Por ello las publicaciones originales para la determinación de fibra (FND y FAD) están obsoletas. Además de FND, nuevos métodos mejorados para fibra dietética total y polisacáridos no almidonosos, incluidos la pectina y $\beta$-glucan están ahora disponibles. A diferencia del almidón, la fermentación de estos polisacáridos en el rumen, es como la de la celulosa, pero más rápida y no genera ácido láctico.

\section{Análisis de costos}

La determinación de costos en el presente estudio se hizo sobre la base de 600 bolsas de $45 \mathrm{~kg}$ (27 t), elaboradas en su momento para dos experimentos, el presente de determinación de la calidad y otro para un estudio de respuesta animal. Para ello se necesitaron 5 jornales durante dos días, lo cual incluyó el enfardado, aplicación de la urea, carga y descarga de las bolsas; el costo del jornal actual es de $\varnothing 7000,00$. El costo de la pulpa es el del transporte de la planta en Santa Cecilia de la Cruz, Guanacaste, en este caso, a la Estación Experimental en Cañas, la planta no hace ningún cargo monetario por la pulpa. El costo de transporte de una traileta, con capacidad de 30 t de pulpa y puesta en Cañas, a precios de hoy, es de $\$ 275000,00$. Otros costos actualizados son el saco de urea de $46 \mathrm{~kg}$ es de $\varnothing 35$ 595,00; el de la bolsa de polietileno, cuyas dimensiones se mencionaron en el capitulo de materiales y métodos del presente estudio, la cual viene en bobinas de aproximadamente 700 bolsas, tiene un precio actualizado de $₫ 160$ cada una; similar al que se consiguen los sacos de propileno cuando se compran pacas de 500 a más sacos. El rubro de otros incluye la piola, tarjetas y marcadores, mano de obra almacenamiento y otros, calculado sobre la base de un 9,2\% del costo de todo lo anterior.

Para la determinación del costo en base fresca o base seca se utilizaron los rubros enumerados en el Cuadro 2. 
Morales et al.:Evaluación de la Calidad y el Costo del Ensilaje de Pulpa de Naranja elaborado en Bolsas de Polietileno1

Cuadro 2. Costos de producción en base seca y fresca del ensilaje de pulpa de naranja. Guanacaste, Costa Rica. 1999.

\begin{tabular}{cc}
\hline Rubro & Costo $\boldsymbol{\varnothing}$ Unitario \\
\hline Bolsa de polietileno & 160,00 \\
Saco propileno & 160,00 \\
Urea $150 \mathrm{~g}$ & 116,00 \\
Transporte & 412,65 \\
Mano de obra & 117,00 \\
Otros & 88,80 \\
Total & 1054,45 \\
Kg ensilaje fresco & 23,43 \\
Kg ensilaje base seca & 137,84 \\
\hline
\end{tabular}

Tasa cambio $\$ 1=\varnothing 515,00$

Como se puede observar en el cuadro anterior, el costo del $\mathrm{kg}$ de ensilaje de pulpa de naranja con $150 \mathrm{~g}$ de urea fue de $\varnothing 23,43$ en base fresca y de $\varnothing 137,84$ en base seca (17\% promedio de materia seca); esto a precios actualizados a octubre del 2010.

\section{CONCLUSIONESY RECOMENDACIONES}

El procesamiento de ensilaje de pulpa de naranja en bolsas de polietileno, tal como se hizo en el presente estudio, resulta un método muy práctico y efectivo. Las características físicas y contenido de azúcares naturales de este subproducto de la industria extractora de jugo de cítricos, lo hacen un material muy fácil de ensilar, ya que no es problema alcanzar las condiciones anaeróbicas, ni el contenido necesario de carbohidratos, para que se active el proceso y se obtengan las condiciones, que hacen del ensilaje, un medio idóneo de conservación de alimentos para animales.

Esto queda demostrado, por el hecho de que hasta los 360 días, prácticamente se mantuvieron las características nutricionales que presentó el ensilaje a los 30 días. Lo anterior tiene dos significados, a los 30 días el proceso de ensilado se ha alcanzado y se conserva así, por lo menos hasta el año, es decir podría ser utilizado por los animales aún a 360 días después de almacenado.

El ensilaje de pulpa de naranja, elaborado en bolsas de polietileno y al cual se le agrega urea en dosis de $150 \mathrm{~g}$ por cada $45 \mathrm{~kg}$ de pulpa, presentó contenidos de proteína cruda sobre el $11 \%$ y características organolépticas relacionadas con el olor, color y textura deseables en un material conservado en buen estado, aún a 360 días de elaborado.

La bolsa plástica de 5 micras de grosor, $110 \mathrm{~cm}$ de largo y $60 \mathrm{~cm}$ de ancho resultó suficiente para obtener un buen ensilaje de pulpa de naranja, como se indicó anteriormente. Las características físicas y el tipo de carbohidratos de la pulpa, permitieron con simples sacudidas de la bolsa plástica, la compactación necesaria para alcanzar condiciones anaeróbicas mínimas para el ensilado. De tal manera que el cierre o amarre de la boca de las bolsas plásticas, aportó poco a este proceso y si mucho a mantener el contenido dentro de la bolsa. El saco de propileno contribuyó grandemente a la manipulación de los sacos y al mantenimiento de la bolsa plástica y su contenido en el tiempo, hasta los 360 días. A los 540 días de elaborado el ensilaje, las pérdidas por daños de ratas fueron casi totales, en donde tanto el saco, como la bolsa fueron rotos, por lo que el ensilaje sufrió el deterioro por efecto de la entrada de aire, pudriéndose todo el contenido.

A precios de octubre del 2010, el costo del proceso de ensilado de pulpa de naranja con $150,00 \mathrm{~g}$ de urea fue de $\phi 23,43$ en base fresca y de $\varnothing 137,84$ en base seca. Comparado con otros alimentos para bovinos de similar contenido nutricional, tal como el maíz que contiene 3,80 Mcal Energía Digestible (ED)/ $\mathrm{kg}$ (MS), el cual tiene un precio aproximado de $\varnothing 60,00$ en base fresca (Morales 2008), el ensilaje de pulpa de naranja se presenta como una opción para la alimentación de bovinos en Costa Rica, máxime que podría comercializarse y almacenarse como se indica en el estudio. 
El bajo contenido de materia seca, tanto de la materia prima utilizada como del producto obtenido en el proceso del ensilaje, permanecen como las partes débiles de este producto. Sin embargo, quedan pendientes evaluaciones, que al igual que la adición de urea, puedan probar la adición de algunos materiales que aporten al mejoramiento del contenido de proteína cruda, pero también al contenido de materia seca (Gohl sf), tal como sería el caso de la adición de alguna leguminosa como, Arachis pintoi, Cratylia argentea, entre otras.

\section{LITERATURA CITADA}

AOAC (Association of Official Agricultural Chemists). 1980. Official methods of Analysis (13 edition). Assoc. Off. Anal. Chem.; Washington, D.C.

Araya G., J. 2001. Acciones de prevención contra la leprosis de los cítricos en Costa Rica. Revista Manejo Integrado de Plagas. (62): 8.

Ashbell G. and Weinberg Zwi G. sf. Silage prodution and utilization. Artículo en línea consultado el 15 de febrebro 2008. Disponible en http:// www.fao.org/ag/AGP/AGPC/doc/ silage/silage_israel/silege_israel. htm. sp.

Ashbell, G., T. Kipnis, M. Titterton, Y. Hen, A. Azrieli y Z. G. Wienberg. 2001. Examination of a technology for silage making in plastic bags. Animal Feed Science and Technology. The Netherlands. 91(3-4): 213-222.

Blazinger, S.B. 2003. Cattle Today: feed supplements come in several different forms. Artículo en línea consultado el 17 de febrero del 2008. USA. Disponible en http://www. Catle today.com/ archive/2003/February/CT251. shtml-16ф-17feb2008. 4 p.
Braddock, R.I. 1999. Handbook of citrus by-products and processing Technology.Wiley and Sons Inc. NY. USA. 247 p.

Chapman, H.L., C.B. Ammerman, F.S. Baker, J.F. Hentges, B.W. Hayes, T.J. sf.Cunha. Citrus Feeds for Beef Cattle. Anim Sci. Dep. Coop. Ext. Serv. IFAS. University of Florida. USA. Artículo en línea consultado el 19 de oct. 2007. Disponible en EDIS Web Site at http:// edis.ifas.ufl.edu $6 \mathrm{p}$.

Citrech. Sf. Structure of citrus fruits. 2008. Italy. Artículo en línea consultado el 11 de marzo del 2008. Disponible en www.citrech.it/English/Information. $\mathrm{htm}$.

Fraser, M.D.; Fychan, R.; Jones, R. 2004. Evaluation of methods for storing small quantities of experimental silage. The Netherlands. Small Ruminant Research 54 (1-2): 141146.

Getachew Gebru, Beyene Chichaibelu and Jess D. Reed. 2005. Laboratory evaluation of the effects of processing methods, treatment snd coffee cultivar on chemical composition and in vitro digestibility of coffee pulp. Disponible en http://www.fao.org/Wairdocs/ILRI/ x5536E/x5536e18.htm. 11 p.

Goering, H. K. and van Soest, P. J. 1970. Forage fibre analysis. Agricultural handboock № 379 . USDA, Washington, D.C.

Gohl, B.I. sf. Citrus by-products for animal feed. In: FAO Ruminant nutrition: selected articles from the World Animal Review. 1978. Italy. Artículo en línea consultado el 19 de octubre. 2007. Disponible en http://www.fao.org/ DOCREP/004/X6512E/X6512E08. htm. $5 \mathrm{p}$. 
Jung, H. J. 1997. Analysis of forage fiber and cell walls in ruminant nutrition. J. of Nutr. 127 (5): 810S-813S. USA.

Lane, I.R. 2000. Poster 5.1: Little bag silage - lan R. Lane. In: Silage making in the tropics with particular emphasis on smallholders. Proccedings of the FAO Electronic Conference on tropical Silage 1999. L. 't Mannetge (ed.) Rome. Italy. Artículo en línea consultado el 18 de febrero 2008. Disponible en http://www.fao.org/ docrep/005/x8486e/x8486e0k.htm.

Loosli, J.K. and I.W. Mcdonald. 1968. Nonprotein nitrogen in the nutrition of ruminants. FAO Agricultural Studies № 73. Rome, Italy. Localizado en internet www.fao.org./docrep/004/ acl149e/AC149E00.HTM el 10/03/2008. $10 \mathrm{p}$.

Mertens, D.R. 2003. Challenges in measuring insoluble dietary fiber. J. Anim. Sci. 81: 3233-3249. USA.

Minson, D. J. 1981. Effects of chemical and physical composition of herbage eaten upon intake. In: Nutritional limits to animal production from pastures. J.B. Hacker (Ed.) Commonwealth Agricultural Bureau. CSIRO. Australia. p $167-182$.

Mohnen, D. sf. Plant Cell Walls. Artículo en línea consultado en febrero del 2008. USA. Disponible en www.ccrc. uga.edu/ dmohnen/bcmb8020/ Plant Wall-06 page.pdf $26 \mathrm{p}$.

Mohnen, D. 2007. Research on the plant cell wall polysaccharide pectin leads to the identification of a gene family tat impacts plant biomass and plant based products. NRI Research Highligths. № 3, 10 p. USDA. Artículo en línea consultado en abril del 2008. Disponible en http:// www.csrees.usda.gov/nri.
Moran, J. 2005. Making quality silage. Tropical dairy farming: feeding management for small holder dairy farmers in the tropics. UK. Lanlinks Press. p $83-97$

Morales, J. L. 1989. Managing the plantanimal interface in tropical legumegrass pastures. Ph.D. Dissertation. USA. University of Florida, Florida.

, R.; Bejarano, Acuña, V. y Castro, M.V.. 2005. Desarrollo de una curva de calibración para analizador de espectrofotometría infrarroja cercana (NIRS) del heno de maní forrajero INTA-Falconiana asociado a pasto transvala (Digitaria decumbens CV. Transvala) para la determinación rápida de su contenido nutricional, que servirá de base para la certificación en los procesos de mercadeo del producto. Informe. INTA, Costa Rica. $11 \mathrm{p}$.

-----, J.L., Cruz, A. y Acuña, V.. 2006a. Efecto del estado de madurez y la fertilización nitrogenada sobre la producción y valor nutritivo del pasto transvala (Digitaria decumbens cv. Transvala) para henificación bajo condiciones de secano. Costa Rica. Alcances Tecnológicos. 4 (1): $37-$ 44.

-------, J.L., V. Acuña y A. Cruz. 2006b. Efecto de la fertilización nitrogenada sobre la producción y valor nutritivo del pasto transvala (Digitaria decumbens cv. Transvala) para henificación bajo condiciones de riego. Costa Rica. Alcances Tecnológicos. 4(1): 45-51.

-------, J.L.; Cruz, A. y Acuña, V. 2001. Uso de la pulpa de naranja en ensilajes comerciales. San José, Costa Rica. Revista Montecillos. Año XVII. (111). 2 p. 
-------, J.L.; Acuña, V. y Cruz, A. 2004. El uso del ensilaje de pulpa de naranja en el engorde de toretes estabulados. Resúmenes XLLX Reunión. PCCMCA. El Salvador. 1 p.

NRC. (National Research Council). 2001. Nutrient Requirements of Dairy cattle: Seventh Revised Edition. USA $157 \mathrm{p}$.

Oluremi, I., Ngi, J. y Andrew, I. A. 2007. Phytonutrients in citrus fruit peal meal and nutritional implications for livestock production. Livestock Research for Rural Development 19(7). Colombia. 5 p. Artículo en línea consultado el 20 de febrero 2008. Disponible en http:// www.cipav.org.co/Irrd/Irrd19/7/ olvr19089.htm.

Reed, J.D. and Van Soest, P.J. 1985. Estimating the nutritive value of crop residue and agro-industrial by-products in animal feeding by chemical methods. In: Better utilization of crop residues and by products in animal feeding: research guidelines 1. State of knowlage. FAO. Italy. $18 \mathrm{p}$.

Sunvold, G.D.; Hussein, H.S.; Fahey Jr., G.C., Merchen, N.R. y Reinhart. G.A.. 1995. In Vitro fermentation of cellulose, beep pulp, citrus pulp and citrus pectin using fecal inoculum from cats, dogs, horses, humans and pigs and ruminal fluid from cattle. USA. J. Anim. Sci. (73): 3639-3648.

Van Soest, P.J. 1963. Use of detergents in the analysis of fibrous feeds. II. A rapid method for the determination of fiber and lignin. USA. J. Assoc. Office Anal. Chem. (46): 289.
Van Soest, P.J.; Robertson, J. B.; and Lewis, B. A. 1991. Methods for dietary fiber, neutral detergent fiber and no starch polysaccharides in relation to animal nutrition. USA. J. Dairy Science. 74(10): 3583-3597.

Weiss, B. 2008. Silage additives. AGF-01892. Agronomy Facts. Ohio State University. Documento en línea consultado el 15 de febrero del 2008. USA. Disponible en http:// ohioline.osu.edu/agf.fact/0018.htm

Wing, J.M (ed). 2003. Citrus feedstuffs for Dairy Cattle. Bul 829. Anim Sci. Dep. Coop. Ext. Serv. IFAS. 1982. Publication Reviewed 2003. USA. Artículo en línea consultado el 19 de octubre, 2007. Disponible en EDIS Web Site at http://edis.ifas. ufl.edu. 